\title{
Autopercepção do ponto de constrição da língua nas fricativas alveolares em mulheres jovens com posicionamento de língua normal e alterado
}

\author{
Self-perception of tongue tip constriction in \\ alveolar fricatives produced by young women \\ with and without normal tongue positioning
}

Descritores

Fala

Percepção da Fala

Língua

Adulto

Fonética

\section{Keywords Speech}

Speech Perception Tongue

Adult

Phonetics

Endereço para correspondência: Viviane Cristina de Castro Marino Universidade Estadual Paulista "Júlio de Mesquita Filho" - UNESP Av. Hygino Muzzi Filho, 737, Bairro Mirante, Marília (SP), Brasil, CEP. 17525-900.

E-mail: vivianemarino2@yahoo.com.br

Recebido em: Abril 17, 2020

Aceito em: Outubro 20, 2020

\section{RESUMO}

Objetivo: Investigar a autopercepção do ponto de constrição da língua na produção de $[\mathrm{s}] \mathrm{e}[\mathrm{z}]$ por jovens monolíngues com posicionamento de língua normal e alterado. Método: Três fonoaudiólogas analisaram gravações de fala em vídeo de 49 mulheres, com idades entre 18 e 28 anos (média=20 anos e 7 meses), classificando-as em: Grupo 1, (G1, n=25), com ausência de alterações no posicionamento de língua na produção de [s] e [z] e Grupo 2 (G2, n=24), com alterações no posicionamento de língua nestes fones. A autopercepção do ponto de constrição da língua foi investigada questionando cada jovem sobre o local em que sua ponta da língua encostava (apical, laminal ou "outros ajustes") ao produzir [s] e [z] na leitura de palavras e pseudopalavras. O teste de Friedman, com comparações par a par posteriores, foi utilizado para análise intragrupo. O teste de MannWhitney foi utilizado para as comparações entre grupos. Adotou-se nível de significância de 5\%. Resultados: Em G1 houve relatos de ponto de constrição apical e laminal enquanto em G2 houve estes relatos, e também de outros ajustes de língua. A presença de outros ajustes da língua diferenciou G1 e G2 (p=0,002). Em G1, houve diferença significativa entre $[\mathrm{s}]$ e $[\mathrm{z}]$, sendo que o ponto laminal ocorreu com mais frequência em [s] do que em $[z]$. Conclusão: Jovens com posicionamento de língua normal e alterado relataram pontos de constrição da língua apical e laminal em fricativas alveolares. Porém, outros ajustes de língua foram percebidos diante de posicionamento de língua alterado.

Trabalho realizado no Laboratório de Análise Articulatória-acústica, Faculdade de Filosofia e Ciências, Universidade Estadual Paulista "Júlio de Mesquita Filho" - UNESP - Marília (SP), Brasil.

${ }^{1}$ Universidade Estadual Paulista "Júlio de Mesquita Filho" - UNESP - Marília (SP), Brasil.

${ }^{2}$ Georgia State University - Atlanta (GA), United States.

${ }^{3}$ Universidade de São Paulo - USP - Bauru (SP), Brasil.

${ }^{4}$ Universidade de Marília - UNIMAR - Marília (SP), Brasil.

Fonte de financiamento: nada a declarar.

Conflito de interesses: nada a declarar. 


\section{INTRODUÇÃO}

A produção da fala é altamente dependente da configuração assumida pela língua no trato vocal. Os sons fricativos alveolares, em particular, requerem posicionamento preciso da língua e da mandíbula, a fim de formar uma constrição estreita na região alveolar e direcionar o fluxo de ar de encontro aos incisivos ${ }^{(1)}$. Especificamente, a produção do fone [z] envolve constrição do fluxo de ar na região alveolar enquanto as pregas vocais permanecem aduzidas para o vozeamento; já a produção do fone [s] requer constrição na região alveolar e abdução das pregas vocais (desvozeamento) ${ }^{(2)}$. Ao realizar constrição, o padrão de contato da língua no palato e o sulco na linha média da língua podem variar entre [s] e [z], conforme observado em estudos envolvendo eletropalatografia ${ }^{(3-7)}$.

A posição que a porção anterior da língua (ponta elevada versus ponta abaixada) assume ao realizar a constrição durante a produção das fricativas alveolares é motivo de discussão na literatura, conforme se observa em estudos envolvendo sujeitos com fala adequada ${ }^{(8,9)}$, alterada (glossectomizados) ${ }^{(1)}$ ou que fazem uso de segunda língua ${ }^{(10)}$. Em geral, recursos instrumentais são usados para fornecer informações sobre a região anterior da língua na produção de $[\mathrm{s}]^{(1,8-11)}$.

Evidências sobre variações no ponto de constrição da ponta da língua na produção de [s] vêm sendo apresentadas desde a década de 90, para falantes do inglês e do francês, com base em achados de palatogramas e linguogramas ${ }^{(8,9)}$. Registros de constrição apical (em que a ponta da língua faz contato parcial com a região alveolar) e laminal (em que a lâmina da língua se eleva enquanto a ponta da língua vai em direção aos dentes inferiores) foram documentados para [s] por meio desse recurso instrumental para falantes do inglês ${ }^{(9)}$. Embora a constrição laminal tenha ocorrido em maior frequência nas produções de [s] de falantes do francês, a constrição apical também foi observada nas produções destes falantes.

A partir de imagens de ressonância magnética, um estudo confirmou a variabilidade na posição da língua na produção de [s] para falantes nativos do inglês (grupo controle), com uso da variante apical por $55 \%$ desses falantes ${ }^{(1)}$. Imagens ultrassonográficas da língua obtidas para nativos do japonês indicaram predominância de ponto de constrição laminal em [s] para esses falantes ${ }^{(10)}$. Imagens ultrassonográficas da língua para falantes do Português Brasileiro mostraram ponta e lâmina da língua anteriorizada, com ou sem ausência de sulco na linha média durante a produção de $[\mathrm{s}]^{(12)}$, o que sugere projeção da porção anterior da língua para cima ou para baixo na produção de [s] por esses falantes. De forma geral, estudos envolvendo recursos instrumentais sugeriram variabilidade no ponto de constrição da língua nas fricativas alveolares entre as línguas e interfalantes.

O método impressionista, que se apoia em auto relatos de falantes, também foi proposto na literatura como uma alternativa para a obtenção de informações sobre ponto de constrição da língua (apical ou laminal) na produção de $[\mathrm{s}] \mathrm{e}[\mathrm{z}]^{(2,13)}$. Com base nas informações derivadas desse método, a prevalência do ponto de constrição da língua em [s] e [z] foi estimada para uma ampla amostra de falantes do hebreu ${ }^{(2)}$. A maioria (60\%) desses falantes relatou autopercepção de uso de ponto de constrição laminal para as fricativas alveolares. Especificamente, o ponto de constrição laminal foi mais comum em [s], prevalecendo uso de ponto de constrição apical em [z]. O estudo apontou para a necessidade de fonoaudiólogos estarem atentos quanto a possibilidade de dois pontos de constrição da língua (apical e laminal) nas fricativas alveolares em falantes do hebreu, sugerindo a adoção de prática individualizada, em que a posição da língua selecionada vá de encontro com a particularidade de cada paciente ${ }^{(2)}$. Em estudo anterior, a variabilidade de ponto de constrição da língua na produção de [s] também foi constatada para falantes nativos do inglês, usando essa mesma metodologia ${ }^{(13)}$, o que suscitou discussões posteriores sobre a necessidade de considerar tais variações no ensino do inglês como segunda língua ${ }^{(10)}$.

De forma geral, os achados obtidos para sujeitos com fala adequada (sem alterações estruturais) levaram a reflexões sobre suas implicações terapêuticas ${ }^{(2,13)}$. Alguns estudiosos, por exemplo, sugeriram verificar qual é o ponto de constrição de língua mais confortável para o paciente na produção das fricativas alveolares, dentro do que é considerado aceitável, a fim de colaborar no direcionamento do processo terapêutico ${ }^{(2)}$, uma vez que a ênfase em um único ponto de constrição de língua para todos os pacientes pode tornar o processo terapêutico mais lento, desmotivando paciente e terapeuta ${ }^{(13)}$. A motivação dessas reflexões para fins terapêuticos reside no fato de [s] ser um dos sons que comumente encontra-se alterado em falantes de diversas línguas ${ }^{(2)}$, podendo a alteração estar presente inclusive na fala do adulto ${ }^{(14-18)}$.

Considerando a suscetibilidade das fricativas alveolares para alterações e/ou compensações na fala de adultos, para fins clínicos, é de interesse obter informações prévias sobre possíveis variações no ponto de constrição da língua para essas fricativas em falantes de uma determinada língua. A autopercepção do ponto de constrição de língua na produção das fricativas alveolares para falantes do Português Brasileiro ainda não foi documentada, nem para adultas com posicionamento de língua normal (com fala adequada) nem para adultas com posicionamento de língua alterada (como ocorre, por exemplo, quando a língua se encontra baixa, entre os dentes ou de encontro com os dentes, porém sem ultrapassá-los). Informações advindas de auto relatos dessas falantes podem contribuir para o conhecimento do fonoaudiólogo sobre possíveis variações nos pontos de constrição da língua na produção de [s] e [z], trazendo reflexões para sua prática clínica.

O objetivo principal do estudo foi investigar a autopercepção do ponto de constrição da língua na produção de fricativas alveolares [s] e [z] por jovens adultas com posicionamento de língua normal e alterado. Objetivou-se, ainda, verificar diferenças na autopercepção do ponto de constrição entre as fricativas, de acordo com o posicionamento da língua, normal ou alterado, assim como possíveis diferenças entre os grupos, para cada fricativa, em ambas, palavras e pseudopalavras. Especula-se que achados de autopercepção variem tanto para jovens adultas que apresentam posicionamento de língua normal quanto alterado e, principalmente, entre esses dois grupos de jovens. Além disso, levanta-se a hipótese de que haveria diferenças na autopercepção dos pontos de constrição da língua entre $[\mathrm{s}] \mathrm{e}[\mathrm{z}]$ no interior de cada grupo, além de diferenças intergrupos, em cada fricativa, durante a produção de palavras e pseudopalavras.

\section{MÉTODO}

Estudo transversal, observacional, analítico e comparativo que se caracteriza como subprojeto de um amplo estudo, aprovado pelo Comitê de Ética em Pesquisa em Seres Humanos da 
Instituição em que foi realizada a pesquisa ( $\mathrm{N}^{\circ}$. 2.915.882/2018). Participaram do estudo 49 jovens adultas, com idades entre 18 e 28 anos (média=20 anos e 7 meses), falantes do Português Brasileiro, oriundas da região Centro-Oeste do Estado de São Paulo, monolíngues. As jovens foram recrutadas de cursos de graduação de uma instituição de ensino superior caracterizando amostra de conveniência. Todas as jovens assinaram o Termo de Consentimento Livre e Esclarecido.

Para a seleção da amostra, inicialmente as jovens preencheram um questionário para se obter informações quanto a queixas de audição e deglutição, sobre a realização de tratamentos fonoaudiológico e ortodôntico, bem como cirúrgico envolvendo frênulo lingual e cirurgia ortognática, além do uso de prótese dentária; também foi questionado sobre alterações na fala como dificuldade em produzir algum som, velocidade aumentada, presença de gagueira e compreensão da fala por parte do ouvinte. Em seguida, uma fonoaudióloga com experiência na avaliação das alterações miofuncionais orofaciais (primeira autora) realizou uma breve entrevista para confirmar os dados levantados no questionário a respeito de alterações na fala e verificar aspectos gerais de fala, como presença de possíveis alterações de fala e tipos, variações dialetais, bem como saber sobre o conhecimento a respeito do processo de produção dos sons da fala; nesse momento também foi realizada a avaliação do frênulo lingual, conforme proposto pelo protocolo $\mathrm{MGBR}^{(19)}$.

Após essa etapa somente foram selecionadas aquelas jovens que não apresentavam histórico de: cirurgias de ortognática e/ ou de liberação do frênulo lingual, tratamento fonoaudiológico, uso de aparelho ortodôntico ou com retirada deste há mais de dois anos e, no caso de contensão ortodôntica, somente com tempo superior a seis meses; sem queixas e/ou histórico de perdas auditivas e sem alterações na fala, bem como do frênulo lingual. Além disso, somente foram incluídas jovens que não apresentavam conhecimento teórico prévio sobre produção dos sons da fala em condições típicas e desviantes, uma vez que tais questões poderiam interferir na realização do teste proposto e comprometer os resultados.

Por fim, as jovens selecionadas passaram por uma triagem ortodôntica realizada por um ortodontista, que verificou a relação dos arcos dentários e a posição dentária individual, a presença de guia de desoclusão, o perfil e o tipo facial, além de serem questionadas sobre possível quadro de disfunção temporomandibular (DTM). Somente foram incluídas no estudo as jovens que apresentavam oclusão Classe I, segundo a classificação de Angle e que não apresentavam sinais/sintomas de DTM. A presença de alterações dentárias individuais discretas (diastema lateral, apinhamento ou rotação do incisivo lateral inferior, ou cruzamentos de um dente) não foram consideradas.

Após a aplicação dos critérios de inclusão e exclusão, foram realizadas gravações em vídeo de amostras de fala das participantes, a fim de possibilitar posterior análise. Para isso, as participantes responderam às perguntas: $O$ que você gosta de assistir na televisão? O que você gosta de fazer? O que você gosta de comer? Também contaram de 1 a 20 , de 60 a 70 e relataram os dias da semana. Três fonoaudiólogas com pelo menos cinco anos de experiência na avaliação clínica das alterações de fala realizaram, individualmente, a análise das amostras de fala registradas, atentando-se para o posicionamento da língua durante a produção dos fones fricativos alveolares [s] e [z].
Nesse estudo, considerou-se como posicionamento de língua alterado, qualquer ajuste atípico no posicionamento da língua que pudesse ser visualmente identificado pelas avaliadoras, podendo ou não ter prejuízo no correlato perceptivo-auditivo da produção do fone-alvo realizada. Estes ajustes incluíram língua com posicionamento baixo, escape lateral uni ou bilateralmente, língua excessivamente visível lateralmente, porém sem escape de ar ou, ainda, presença de ceceio (anterior ou lateral), sendo tais alterações associadas ou não a desvios mandibulares.

As impressões das fonoaudiólogas foram anotadas e os resultados das análises individuais indicaram concordância total $(100 \%$ de concordância) das respostas quanto a presença ou ausência de posicionamento alterado de língua, o que permitiu a formação de dois grupos: com presença ou ausência de posicionamento alterado da língua. Especificamente, com o propósito apenas de favorecer o entendimento dos tipos de ajustes atípicos apresentados pelas jovens na produção do [s] e [z], para os casos em que não houve concordância nesse aspecto, outra análise foi realizada com as três avaliadoras em conjunto, utilizando o recurso slow motion, para se obter resultados consensuais quanto à caracterização dos ajustes atípicos da língua. Foram obtidos resultados consensuais das fonoaudiólogas do aspecto avaliado para todo material apresentado.

A partir da análise das fonoaudiólogas, dois grupos de participantes foram formados: G1, com posicionamento normal de língua (fala adequada) durante a produção das fricativas alveolares (25 participantes) e G2, com posicionamento de língua alterado durante a produção das fricativas alveolares (24 participantes), com ou sem prejuízo no correlato perceptivo da produção do fone-alvo realizada resultante de tal posicionamento. Uma análise mais detalhada mostrou que no grupo com posicionamento de língua alterado $(n=24)$, a maioria $(87 \%, n=21)$ apresentava alterações sutis que resultavam, na maioria das vezes, em prejuízo no correlato perceptivo da produção do fone-alvo. Mais especificamente, 12 apresentavam escape da língua ( 9 bilateral; 2 unilateral; 1 anterior), 6 apresentavam região lateral da língua visível em excesso (sem escape de ar), 2 apresentavam língua em posição baixa, sendo estas alterações acompanhadas ou não de desvio/anteriorização mandibular enquanto uma apresentava língua em posição baixa e região lateral visível em excesso. Somente três participantes apresentavam ceceio, sendo duas ceceio anterior e uma ceceio lateral, conforme descrições da literatura ${ }^{(20)}$.

Os procedimentos realizados neste trabalho foram baseados em descrições de estudo prévio ${ }^{(2)}$ e envolveram duas etapas: treino e teste propriamente dito. Previamente ao início do experimento confirmou-se, por meio de um breve questionário, ausência de relatos de qualquer condição não usual nas estruturas orais (aftas, por exemplo) ou no modo de respirar, que pudessem interferir nas etapas do experimento.

\section{Etapa treino}

Cada participante recebeu, individualmente, uma breve explicação sobre como são produzidos os sons da fala, abordando-se, particularmente, as características de ponto articulatório dos fones [m], [1] e [k]. Para isso, a avaliadora demonstrou o ponto articulatório desses fones (bilabial, alveolar e velar, respectivamente), utilizando material em Power Point, 
apresentado em um notebook. O material contemplava figuras ilustrativas dos articuladores (lábios, língua e palato) envolvidos na produção dos fones alvos, com apresentação simultânea de produções vídeo-gravadas dos respectivos fones. Após a explicação, foi solicitado a cada participante que lesse cada uma das seis palavras apresentadas individualmente na tela do computador. As palavras eram constituídas pelos fones [1], [m], [k], em posição inicial ou medial (Lata, Tala, Mapa, Puma, Carro, Rico). Após a leitura de cada palavra, a participante deveria relatar os movimentos dos articuladores envolvidos na produção dos fones alvos. A avaliadora ofereceu feedback sobre erros ou acertos após respostas apresentadas pela participante na fase treino. Somente após a participante relatar corretamente quais eram articuladores e seus respectivos movimentos durante a produção dos sons alvos seguiu-se com a fase teste.

\section{Etapa teste}

Para a realização da etapa teste, um total de 48 estímulos de fala foi selecionado, sendo 24 palavras dissílabas e 24 pseudopalavras com a mesma estrutura fonológica das palavras (Quadro 1). Os estímulos de fala apresentavam os fones [s] e [z] nas posições de onset (inicial e medial) ou coda (medial). Os sons que antecediam ou precediam os fones alvos eram alveolares ou não-alveolares, a fim de controlar efeito de coarticulação. A etapa teste foi realizada logo após o treino, apresentando-se, individualmente, a cada participante, os 48 estímulos de fala previamente aleatorizados, em slides padronizados (Power Point), utilizando notebook. Os slides contemplavam os estímulos de falas com os fones alvos em destaque, a fim de favorecer a atenção da participante para estes fones.

As participantes foram orientadas a ler cada estímulo de fala em voz alta, atentando-se à produção do fone alvo e, em seguida, relatar onde a ponta de sua língua encostava ao produzir o fone. Em caso de dúvidas, as participantes poderiam repetir a tarefa, até se sentirem seguras sobre sua resposta. Em nenhum momento foi oferecido qualquer tipo de "feedback" sobre a acuracidade da produção ou da resposta dada. Todas as produções e respostas dadas por cada participante foram filmadas para confirmação posterior dos achados, caso fosse necessário. Os relatos obtidos foram classificados em três tipos de pontos de constrição da língua na produção de [s] e [z]: (a) apical (ponta da língua na região do alvéolo superior); (b) laminal (ponta da língua atrás do alvéolo inferior) e "outros ajustes" (qualquer outro posicionamento da ponta da língua). Uma mesma participante poderia relatar o uso de um único ponto de constrição da língua (somente apical, somente laminal) no total de suas produções ou, ainda, relatar mais de um ponto de constrição (apical, laminal, ambos ou outros ajustes) no total de suas produções.

\section{Forma de análise dos resultados}

A distribuição absoluta e relativa de participantes por ponto de constrição de língua (laminal, apical ou "outros ajustes") em cada grupo (G1, com posicionamento de língua normal e G2, com posicionamento alterado) foi inicialmente obtida. Do total de 1.200 relatos (25 participantes x 48 estímulos) esperados para G1, determinou-se qual era a ocorrência de cada tipo de ponto de constrição de língua. Da mesma forma, do total de 1.150 relatos ( 24 participantes x 48 estímulos) esperados para $\mathrm{G} 2$, estabeleceu-se qual era a ocorrência de cada tipo de ponto de constrição de língua. A média da porcentagem de ocorrência de relatos de autopercepção de cada ponto de constrição de língua (laminal, apical ou "outros ajustes") foi então calculada para cada grupo, considerando as seguintes variáveis: total de amostras de fala $(n=48)$, amostras de fala constituídas por palavras com $[\mathrm{s}](\mathrm{n}=12)$ e $[\mathrm{z}](\mathrm{n}=12)$ e amostra de fala constituídas por pseudopalavras com $(n=12)$ e $[\mathrm{z}](\mathrm{n}=12)$. As comparações das médias obtidas intra e intergrupos foram analisadas por testes estatísticos.

A distribuição de normalidade foi analisada pelo teste de Shapiro-Wilk e mostrou distribuição não paramétrica. Para comparação de medidas repetidas para os relatos dos diferentes pontos de constrição da língua (apical, laminal e "outros ajustes”) em um mesmo grupo de participantes foi realizado o teste de Friedman, com comparações par a par pelo teste de Wilcoxon com correção post-hoc de Holm-Sidak. As comparações entre dois grupos independentes foram realizadas pelo teste de MannWhitney. O nível de significância adotado foi de $5 \%(\mathrm{p} \leq 0,05)$ e os dados foram analisados no software SPSS (versão 24.0).

Quadro 1. Palavras e pseudopalavras com fones [s] e [z], na posição de onset (inicial e medial) e coda (medial)

\begin{tabular}{|c|c|c|c|c|c|c|c|c|c|c|c|}
\hline \multirow{8}{*}{$\frac{\stackrel{0}{\pi}}{\frac{\pi}{\pi}}$} & \multirow{8}{*}{ 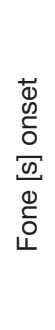 } & \multirow{8}{*}{ 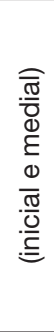 } & SAPA & \multirow{8}{*}{$\frac{\mathscr{N}}{\overbrace{\pi}^{\pi}}$} & \multirow{8}{*}{ 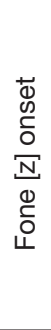 } & \multirow{8}{*}{ 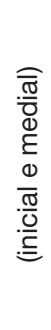 } & ZAPI & \multirow{4}{*}{ 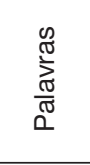 } & \multirow{4}{*}{ 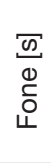 } & \multirow{4}{*}{ 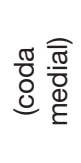 } & \multirow{3}{*}{\begin{tabular}{|l} 
CASPA \\
MASCA \\
VASTA \\
\end{tabular}} \\
\hline & & & SACA & & & & ZAGA & & & & \\
\hline & & & SALA & & & & ZARA & & & & \\
\hline & & & SINA & & & & ZELO & & & & BASTA \\
\hline & & & PASSA & & & & PISA & & & & ASMA \\
\hline & & & CAÇA & & & & CASA & $\stackrel{\pi}{\Sigma}$ & $\frac{N}{\omega}$ & $\frac{\pi}{0} \cdot \widehat{\frac{\pi}{0}}$ & RASGO \\
\hline & & & LAÇA & & & & NASA & $\frac{\pi}{\pi}$ & రั๊ & & \begin{tabular}{|l|} 
ASNO \\
\end{tabular} \\
\hline & & & NASCE & & & & LESE & & & & ROSNA \\
\hline & & & SAPI & & & & ZAPU & & & & COSPA \\
\hline & & & SICU & & & & ZAGUI & 음 윤 & $\frac{\sigma}{0}$ & $\frac{\pi}{0} \cdot \stackrel{\widehat{\widetilde{0}}}{\bar{\pi}}$ & MUSCA \\
\hline$\sum_{\pi}^{\pi}$ & 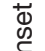 & $\frac{\bar{\sigma}}{\overline{0}}$ & SALI & 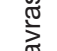 & $\begin{array}{l}\vec{\Phi} \\
\infty\end{array}$ & $\frac{\bar{\sigma}}{\overline{0}}$ & ZARRU & $\underset{\infty}{0} \frac{\pi}{\pi}$ & రั & & \begin{tabular}{|l|} 
VUSTA \\
\end{tabular} \\
\hline$\frac{\pi}{\pi}$ & రิ & $\stackrel{\oplus}{E}$ & SINI & $\frac{\pi}{\pi}$ & ઠ & $\stackrel{\oplus}{E}$ & ZELI & & & & BUSTA \\
\hline 음 & $\stackrel{0}{0}$ & $\frac{0}{\pi}$ & PUSSA & 음 & $\stackrel{N}{0}$ & $\frac{0}{\sigma}$ & PUZA & & & & OZMA \\
\hline गु & ธั & $\frac{.0}{\frac{O}{C}}$ & CUÇA & ఫ్ర & ర్ & $\frac{.0}{\frac{0}{C}}$ & CUZA & 음 꾼 & $\frac{N}{N}$ & $\frac{\pi}{0} . \widehat{\frac{\mathbb{\sigma}}{\pi}}$ & RUZGO \\
\hline & & & LUÇA & & & & NUZA & $\frac{0}{\infty} \frac{\pi}{\pi}$ & రั้ & 过 & \begin{tabular}{|l|} 
UZNO \\
\end{tabular} \\
\hline & & & $\mathrm{NUCl}$ & & & & LUZE & & & & RUZNA \\
\hline
\end{tabular}




\section{RESULTADOS}

A distribuição absoluta e relativa de participantes por ponto de constrição de língua (laminal, apical ou “outros ajustes") em cada grupo é apresentada na Tabela 1. Os achados mostram que jovens do G1 relataram uso de ponto de constrição laminal ou apical enquanto jovens do G2 relataram, além dos pontos apical e laminal, autopercepção de "outros ajustes" de língua durante a produção das fricativas alveolares.

A Tabela 2 mostra a comparação das médias das porcentagens de ocorrência dos relatos de autopercepção para os pontos de constrição laminal, apical ou "outros ajustes" de língua, dentro de cada grupo. Foram observadas diferenças significativas entre os pontos de constrição, tanto para G1 $(\mathrm{p}<0,001)$ quanto para $\mathrm{G} 2(\mathrm{p}<0,001)$, ao produzir as fricativas alveolares. Em ambos os grupos, não houve diferenças entre os pontos de constrição apical e laminal, porém ambos apresentaram diferenças significativas em relação a categoria "outros ajustes" (sem ocorrência). Ao comparar os grupos dentro de cada tipo de constrição, foi observada diferenças significativas somente na categoria "outros ajustes" ( $p=0,002)$, sugerindo que esta categoria de resposta diferenciou os grupos.

Para o estímulo "palavras" houve diferença entre as médias na categoria de resposta "outros ajustes" de língua, ao comparar os grupos (G1 vs. G2) tanto em $[\mathrm{s}](\mathrm{p}=0,004)$ quanto em $[\mathrm{z}]$ $(\mathrm{p}=0,004)$ (Tabela 3).

Para o estímulo "pseudopalavras", também houve diferença entre as médias para a categoria de respostas "outros ajustes" de língua, ao comparar os grupos (G1 vs G2) tanto em $[\mathrm{s}](\mathrm{p}=0,017)$ quanto em $[\mathrm{z}](\mathrm{p}=0,008)$. Houve, ainda, diferença entre $[\mathrm{s}] \mathrm{e}[\mathrm{z}]$ para o ponto de constrição laminal $(p=0,032)$, em que se observou maior média de ocorrência de ponto de constrição laminal para [s] do que para [z], porém somente para G1 (Tabela 4).

Tabela 1. Distribuição de participantes de acordo com ponto de constrição da língua (normal, apical ou ambos) nos grupos com posicionamento de língua normal $(n=25)$ ou alterada $(n=24)$ na produção de fricativas alveolares

\begin{tabular}{|c|c|c|c|c|}
\hline & Laminal & Apical & Laminal ou Apical & Outros \\
\hline & (Total) & (Total) & (Total) & (Total) \\
\hline G1: Normal $(\mathrm{N}=25)$ & $6(24 \%)$ & $7(28 \%)$ & $12(48 \%)$ & $0(0 \%)$ \\
\hline G2: Alterado $(\mathrm{N}=24)$ & $3(12,5 \%)$ & $3(12,5 \%)$ & $10(42 \%)$ & $8(33 \%)$ \\
\hline
\end{tabular}

Tabela 2. Comparação da média e desvio padrão (DP) da porcentagem de ocorrência de relatos de cada ponto de constrição da língua (laminal, apical ou "outros ajustes") para os 48 estímulos de fala, nos grupos de participantes com posicionamento de língua normal e alterado

\begin{tabular}{|c|c|c|c|c|c|}
\hline \multicolumn{6}{|c|}{ Grupos } \\
\hline & \multicolumn{2}{|c|}{ G1: Normal $(n=25)$} & \multicolumn{2}{|c|}{ G2: Alterado $(n=24)$} & $p$-valor \\
\hline & Total & Média & $\mathrm{DP}$ & Média & DP \\
\hline Laminal & $41,50 a$ & 41,84 & $40,62 a$ & 36,23 & 0,992 \\
\hline Apical & $58,49 a$ & 41,84 & $52,69 a$ & 34,26 & 0,421 \\
\hline Outros ajustes & $0,00 b$ & 0,00 & $6,68 b$ & 11,08 & $0,002^{\dagger}$ \\
\hline $\mathrm{p}$-valor & \multicolumn{2}{|c|}{$<0,001^{*}$} & \multicolumn{2}{|c|}{$<0,001^{\star}$} & \\
\hline
\end{tabular}

† indica diferença significativa entre grupos dentro do mesmo tipo de constrição da língua pelo teste não paramétrico de Mann-Whitney

*Diferença significativa em relação ao ponto de constrição da língua (laminal, apical e outros ajustes) dentro do grupo (posicionamento de língua normal ou alterada) pelo teste não paramétrico de Friedman $(<0,001)$. Letras diferentes $(a, b)$ indicam diferenças significativas dentro do grupo pelo teste não paramétrico de Wilcoxon com correção post-hoc de Holm-Sidak

Tabela 3. Comparação da média e desvio padrão (DP) da porcentagem de ocorrência de relatos de ponto de constrição da língua (laminal, apical ou "outros ajustes") entre [s] e [z], por grupo (posicionamento de língua normal e alterado) e entre grupo, por fricativa alveolar, na produção de palavras

\begin{tabular}{|c|c|c|c|c|c|c|c|c|c|c|c|c|}
\hline \multicolumn{9}{|c|}{ Grupo } & \multicolumn{2}{|c|}{ Grupo } & \multicolumn{2}{|c|}{ Fricativa } \\
\hline & \multicolumn{4}{|c|}{ G1:Normal } & \multicolumn{4}{|c|}{ G2: Alterado } & $\mathrm{N}$ & $A$ & $/ \mathrm{s} /$ & $|z|$ \\
\hline & \multicolumn{2}{|c|}{$/ \mathrm{s} /$} & \multicolumn{2}{|c|}{$|z|$} & \multicolumn{2}{|c|}{ /s/ } & \multicolumn{2}{|c|}{$|z|$} & \multicolumn{2}{|c|}{ /s/ vs./z/ } & \multicolumn{2}{|c|}{ N vs. A } \\
\hline Palavra & Média & DP & Média & DP & Média & DP & Média & DP & $\mathrm{p}$ & $\mathrm{p}$ & $\mathrm{p}$ & $\mathrm{p}$ \\
\hline Laminal & 42,6 & 41,4 & 40,3 & 43,3 & 39,2 & 41,9 & 39,5 & 35,0 & 0,363 & 0,412 & 0,643 & 0,684 \\
\hline Apical & 57,3 & 41,4 & 59,6 & 43,3 & 55,9 & 41,4 & 51,7 & 35,3 & 0,363 & 0,361 & 0,847 & 0,320 \\
\hline Outros ajustes & 0,0 & 0,0 & 0,0 & 0,0 & 4,8 & 9,8 & 8,6 & 18,0 & 0,999 & 0,249 & $0,004^{\dagger}$ & $0,004 \dagger$ \\
\hline
\end{tabular}

† indica diferença significativa entre os grupos (normal vs. alterado) para cada fricativa para um mesmo tipo de constrição de língua pelo teste não paramétrico de Mann-Whitney

Legenda: $\mathrm{N}=$ normal; $\mathrm{A}=$ alterado

Tabela 4. Comparação da média e desvio padrão (DP) da porcentagem de ocorrência de relatos de ponto de constrição da língua (laminal, apical ou "outros ajustes") entre [s] e [z], por grupo (posicionamento de língua normal e alterado) e entre grupo, por fricativa alveolar, na produção de pseudopalavras

\begin{tabular}{|c|c|c|c|c|c|c|c|c|c|c|c|c|}
\hline & \multicolumn{8}{|c|}{ Grupo } & \multicolumn{2}{|c|}{ Grupo } & \multicolumn{2}{|c|}{ Fricativa } \\
\hline & \multicolumn{4}{|c|}{ G1: Normal } & \multicolumn{4}{|c|}{ G2: Alterado } & $\mathrm{N}$ & $A$ & $/ \mathrm{s} /$ & $|z|$ \\
\hline & \multicolumn{2}{|c|}{ /s/ } & \multicolumn{2}{|c|}{$|z|$} & \multicolumn{2}{|c|}{ /s/ } & \multicolumn{2}{|c|}{$\mid z /$} & \multicolumn{2}{|c|}{ /s/ vs. /z/ } & \multicolumn{2}{|c|}{$\mathrm{N}$ vs. $\mathrm{A}$} \\
\hline Pseudopalavra & Média & DP & Média & $\mathrm{DP}$ & Média & $\mathrm{DP}$ & Média & $\mathrm{DP}$ & $\mathrm{p}$ & $p$ & $p$ & $p$ \\
\hline Laminal & 45,0 & 42,1 & 38,0 & 44,2 & 43,4 & 39,4 & $40,2 a$ & 38,6 & $0,032^{\ddagger}$ & 0,972 & 0,984 & 0,782 \\
\hline Apical & 54,9 & 42,1 & 62,0 & 44,2 & 52,7 & 38,0 & $50,3 a$ & 38,7 & 0,032 & 0,346 & 0,708 & 0,302 \\
\hline Outros ajustes & 0,0 & 0,0 & 0,0 & 0,0 & 3,8 & 9,5 & $9,3 b$ & 19,6 & & 0,108 & $0,017^{\dagger}$ & $0,008 \dagger$ \\
\hline
\end{tabular}

$\ddagger$ diferença significativa entre as fricativas dentro do grupo (normal ou alterado) e ponto de constrição de língua pelo teste não paramétrico de Wilcoxon; † indica diferença significativa entre os grupos (normal vs. alterado) para cada fricativa (/s/ e /z/) para um mesmo tipo de constrição de língua pelo teste não paramétrico de Mann-Whitney

Legenda: $\mathrm{N}=$ normal; $\mathrm{A}=$ alterado 


\section{DISCUSSÃO}

O presente estudo investigou a autopercepção do ponto de constrição da língua na produção de [s] e [z], por jovens do sexo feminino falantes do Português Brasileiro, que apresentavam posicionamento de língua normal ou alterado, usando metodologia similar àquela proposta em estudos prévios, ou seja, a partir de informações derivadas de método impressionista ${ }^{(2,13)}$. Informações advindas sobre autopercepção da língua restringem-se aos estudos que investigaram autopercepção da posição habitual de língua no repouso ${ }^{(21)}$, na função de deglutição ${ }^{(22)}$ ou na pronúncia de trigramas ou tetragramas, por populações com condições específicas (usuários de prótese ou com desordens temporomandibulares) ${ }^{(23,24)}$, sem enfocar como a língua se posiciona na produção de fricativas alveolares, em falantes do Português Brasileiro.

Os resultados gerais do estudo mostraram que $48 \%$ das jovens com posicionamento de língua normal relatou uso dos pontos de constrição laminal e apical ao produzir [s] e [z], enquanto o restante relatou uso somente de ponto apical ou laminal. Resultado semelhante foi observado no grupo com posicionamento de língua alterado, em que o uso de pontos de constrição da língua apical ou laminal também foi relatado pela maioria ( $42 \%$ ) das jovens, com uma pequena parcela de relatos de uso exclusivo do ápice da língua $(12,5 \%)$ ou de exclusivo de ponto laminal $(12,5 \%)$. Contudo, diferentemente do grupo com posicionamento normal de língua, em 33\% $(\mathrm{N}=8)$ das jovens com posicionamento alterado de língua foram observados "outros ajustes", como posicionamento da região anterior da língua entre os dentes ou, ainda, de encontro com estes. Além disso, algumas dessas jovens também perceberam os pontos de constrição apical e laminal (25\%), ou apical ( $8 \%$ ) ou laminal (4\%).

Na literatura, a variabilidade de ponto de constrição da língua em fricativas alveolares foi observada para falantes do inglês, em um estudo que constatou que $30 \%$ dos falantes que tinham inglês como primeira língua, usavam posição não alveolar (com a lâmina da língua criando constrição parcial) ao pronunciar [s], enquanto os demais usavam ponto apical ${ }^{(13)}$; assim como para falantes do hebreu, em que $60 \%$ da amostra estudada relatou perceber uso exclusivo de ponto de constrição laminal da língua nas fricativas alveolares, enquanto os demais relataram uso do ponto apical ou, ainda, de ambos ${ }^{(2)}$.

No estudo, a partir da análise de cada ponto de constrição de língua, dentro de cada grupo, não foi verificada diferença entre as médias de ocorrência do ponto de constrição apical e laminal para o jovens com posicionamento de língua normal, e não houve relatos de "outros ajustes" de língua, o que confirma a possibilidade de uso tanto de pontos de constrição apical quanto laminal nos fones fricativos alveolares por jovens falantes do Português Brasileiro, com posicionamento de língua normal, corroborando estudos que também sugerem relatos de ponto de constrição laminal ou apical por falantes de outras línguas ${ }^{(2,13)}$.

Variações discretas nos pontos de contato da língua são esperadas entre sujeitos com fala adequada, já que estes apresentam estruturas faciais e musculaturas diferentes e, portanto, mesmo quando esses pontos não são exatamente os mesmos, o produto final pode ser percebido auditivamente como correto ${ }^{(17)}$. Até mesmo fonoaudiólogos clínicos, falantes do Português Brasileiro, nem sempre identificam facilmente a posição que sua língua assume ao produzir o fone [s] quando questionados, ainda que informalmente, em cursos ou aulas, resultando, muitas vezes, em relatos distintos sobre o ponto de constrição da língua na produção deste fone ${ }^{(17)}$. Variações interfalantes no posicionamento da língua na produção de [s] foram documentadas previamente, com base em achados instrumentais, sugerindo que aspectos como, por exemplo, a altura do palato, pode influenciar a configuração da porção anterior da língua durante a produção de $[\mathrm{s}]^{(1,11)}$. Um estudo ${ }^{(1)}$ verificou que sujeitos com fala adequada que apresentavam palato baixo tenderam a usar ponto apical, enquanto aqueles com palato alto tenderam a usar ponto laminal na produção de [s]. Levanta-se, portanto, a possibilidade de que a variabilidade nos pontos de constrição da língua na produção das fricativas alveolares observada nas jovens com posicionamento de língua normal do presente estudo possa ser decorrente, em parte, de possíveis diferenças na altura do palato dessas jovens.

A análise de cada ponto de constrição de língua também mostrou variabilidade no posicionamento da ponta da língua na produção das fricativas [s] e [z] para jovens com posicionamento de língua alterado, demonstrada pelo uso prevalente dos pontos de constrição apical e laminal, porém, com relatos de "outros ajustes" de língua. Ao questionar sujeitos com alterações de posicionamento de língua durante o processo de avaliação fonoaudiológica espera-se, portanto, relatos de constrição não usuais da ponta da língua na produção de [s] e [z]. Estudo prévio enfatizou a necessidade de levar em consideração os ajustes de língua apresentado por um determinado falante para favorecer planejamento terapêutico ${ }^{(2)}$.

A comparação intergrupos mostrou diferença significativa na média de ocorrência de auto relatos somente para a categoria "outros ajustes" de língua, observado apenas no grupo com posicionamento alterado de língua, confirmando, portanto, que a presença dos relatos de "outros ajustes" de língua diferenciou os dois grupos de jovens. Nota-se, portanto, que posições não usuais mais sutis da língua, e não a presença de ceceio lateral propriamente dito, levaram o restante das jovens a relatarem "outros ajustes" de língua, ao produzir as fricativas alveolares. Embora a presença de ceceio anterior (leve) tenha sido identificada em duas das 24 jovens, estas não relataram "outros ajustes" de língua.

Os dados da avaliação ortodôntica permitiram verificar que nove $(37,5 \%)$ jovens com posicionamento alterado de língua apresentaram alterações dentárias individuais (diastema lateral, apinhamento ou rotação do incisivo lateral inferior) ou cruzamento de um dente, das quais cinco relataram presença de "outros ajustes" de língua ao produzir [s] ou [z], enquanto seis relataram exclusivamente pontos de constrição apical ou laminal. Estes achados sugerem que as alterações individuais nos dentes parecem não ter favorecido a percepção do uso de "outros ajustes" de língua na produção de fones fricativos alveolares. Além disso, uma inspeção mais detalhada da análise das fonoaudiólogas mostrou que das 24 participantes com posicionamento alterado de língua, 8 apresentaram leves desvios mandibulares e, dessas, metade $(n=4)$ apresentou relatos de "outros ajustes" de língua. Os desvios mandibulares, por si só, parecem também não justificar a presença de "outros ajustes" 
de língua relatados pelas participantes com posicionamento alterado de língua.

A posição que a língua assume ao produzir especificamente a fricativa alveolar vozeada ou não vozeada é de interesse para clínicos e pesquisadores ${ }^{(2)}$. Também é de interesse conhecer se a autopercepção do ponto de constrição da língua entre essas fricativas varia em função do estímulo de fala. As comparações intragrupo mostraram que, para as palavras, não houve diferenças entre [s] e [z], diferente dos achados pseudopalavras em que houve diferença significativa entre estes fones, com maior ocorrência de ponto de constrição de língua laminal para [s] do que [z], para as jovens com posicionamento normal de língua. Embora tenha sido observado tendência de maior ocorrência de ponto de constrição de língua apical para $[\mathrm{z}]$ do que $[\mathrm{s}]$, a diferença encontrada não foi significativa. A hipótese de que haveria diferenças entre as fricativas [s] e [z] no interior de cada grupo foi parcialmente comprovada, uma vez que a diferença ocorreu para um dos grupos, somente para pseudopalavras.

$\mathrm{O}$ fato de essa diferença ser significativa apenas em pseudopalavras pode ser justificada pelo maior nível de atenção exigido na tarefa de autopercepção da ponta da língua ao produzir estímulos de fala não usuais, ou seja, estímulos que soam como palavras e são compostos pelas mesmas regras fonológicas, mas que não carregam significado ${ }^{(25)}$. Tal fato pode ter levado às jovens com posicionamento de língua normal a perceberem diferenças sutis da posição da ponta da língua entre [s] e [z], resultando na maior ocorrência de relatos de constrição laminal em [s] do que $[\mathrm{z}]$.

Diferenças entre as fricativas alveolares vozeadas e não vozeadas foram documentadas pela eletropalatografia previamente. Em geral, os achados mostram que a fricativa não vozeada é um pouco menos anterior do que a vozeada ${ }^{(5)}$ com menor contato língua/palato (anterior) do que sua contrapartida vozeada ${ }^{(3-5)}$, com sulco anterior na linha média da língua mais largo em [s] do que [z] enquanto o sulco posterior é mais largo em [z] e mais estreito em $[\mathrm{s}]^{(5)}$. Além disso, o tempo articulatório do padrão de contato da língua com palato difere, com duração de $[\mathrm{s}]$ significativamente mais longa que $[\mathrm{z}]^{(6,7)}$. Essas diferenças entre as fricativas alveolares podem ter favorecido a maior percepção de constrição laminal em [s] do que em [z], porém, em estímulos que requerem maior atenção para a realização de novos movimentos articulatórios (pseudopalavras). Os achados do estudo da produção de /s/e /z/ em pseudopalavras concordaram com aqueles reportados previamente para falantes do hebreu ${ }^{(2)}$, no que diz respeito à produção laminal ser mais frequente em [s] do que [z], porém discordam dos achados ao não observar diferenças significativas na autopercepção do ponto de constrição de $/ \mathrm{s} / \mathrm{e} / \mathrm{z} / \mathrm{em}$ palavras.

No presente estudo ainda foi verificado se os achados se diferenciaram entre os grupos, para cada fricativa, nas palavras e nas pseudopalavras. A hipótese de que haveria diferenças na autopercepção dos pontos de constrição da língua intergrupos, para cada fricativa, em palavras e pseudopalavras, foi parcialmente comprovada. Os resultados mostraram que para ambas fricativas, tanto em palavras quanto em pseudopalavras, houve diferença significativa entre os grupos apenas para a categoria de respostas "outros ajustes de língua", em [s] e [z]. Com base nestes achados pode-se notar que o posicionamento da língua de cada grupo (normal ou alterado) influenciou os resultados, com comportamento similar em [s] e [z], tanto em palavras quanto pseudopalavras.

O foco do presente estudo foi oferecer informações sobre o ponto de constrição de língua na produção de [s] e [z] por jovens adultas com e sem posicionamento normal de língua na produção de fricativas alveolares, com base em achados derivados de método impressionista. Este método é considerado um recurso simples, de fácil aplicação e de baixo custo que pode ser utilizado na clínica fonoaudiológica, por oferecer informações importantes sobre como o paciente percebe os movimentos da parte anterior da língua ao produzir os sons fricativos ${ }^{(2)}$. Espera-se que as informações obtidas no estudo permitam reflexões que possam auxiliar a avaliação fonoaudiológica, direcionando o processo terapêutico.

Há evidências na literatura de que um sujeito pode perceber e, ainda, mensurar variações em sua pronúncia (leitura de trigramas ou tetragramas, ou seja, letras justapostas sem significado), em condições controladas (uso de prótese dentária ${ }^{(23)}$ ou placa de oclusão estabilizadora ${ }^{(24)}$ ) e, portanto, os julgamentos de autopercepção de um paciente podem auxiliar e contribuir para o processo de avaliação fonoaudiológica ${ }^{(24)}$. Espera-se que o teste de autopercepção do ponto de constrição da língua em [s] e [z], conforme proposto no estudo, possa ser usado como um recurso adicional utilizado no processo de avaliação fonoaudiológica, possibilitando constatar auto relatos de ajustes de língua distintos na presença de condições adversas (posicionamento alterado de língua). Para fins clínicos, sugere-se, durante o processo de avaliação, verificar a percepção que o próprio paciente tem em relação ao seu ponto de constrição da língua ao produzir [s] e [z] e, para fins terapêuticos, buscar o ponto de constrição de língua nas fricativas alveolares mais confortável para o paciente ${ }^{(2)}$ que, com base nos achados do estudo, pode ser apical ou laminal, para mulheres adultas.

Algumas limitações do estudo dizem respeito ao restrito número de participantes de cada grupo investigado, ao não controle da gravidade da alteração do posicionamento da língua das participantes e, também, da vogal incluída nos estímulos de fala. Em estudos futuros sugere-se ampliar o número de participantes, investigar população de ambos os sexos, com diferentes tipos de ceceio, além de adicionar informações advindas de recursos instrumentais (ultrassonografia, por exemplo) que possibilitem comparações com achados de autopercepção da língua na produção de [s] e [z].

\section{CONCLUSÃO}

Pontos de constrição de língua apical ou laminal durante a produção dos fones [s] e [z] foram percebidos por jovens adultas com posicionamento de língua normal e não houve diferença desses relatos em palavras, diferentemente de pseudopalavras em que o ponto laminal ocorreu com mais frequência em [s] do que em [z]. Para jovens com posicionamento alterado de língua, não houve diferença para os relatos do ponto de constrição na produção de [s] e [z], tanto em palavras como em pseudopalavras. A comparação dos achados entre os grupos mostrou que jovens com posicionamento de língua normal relataram pontos de constrição apical e laminal, enquanto jovens com posicionamento alterado de língua relataram pontos de constrição apical e laminal, e também outros ajustes de língua. 


\section{REFERÊNCIAS}

1. Stone M, Rizk S, Woo J, Murano EZ, Chen H, Prince JL. Frequency of apical and laminal /s/ in normal and post-glossectomy patients. J Med Speech-Lang Pathol. 2013;20(4):106-11.

2. Icht M, Ben-David BM. Sibilant production in Hebrew-speaking adults: apical versus laminal. Clin Linguist Phon. 2018;32(3):193-212. http:// dx.doi.org/10.1080/02699206.2017.1335780. PMid:28727493.

3. Dagenais PA, Lorendo LC, McCutcheon MJ. A study of voicing context effects upon consonant linguapalatal contact patterns. J Phonetics. 1994;22(3):225-38. http://dx.doi.org/10.1016/S0095-4470(19)30202-5.

4. McLeod S, Roberts A, Sita J. Tongue/palate contact for the production of /s/ and /z/. Clin Linguist Phon. 2006;20(1):51-66. http://dx.doi. org/10.1080/02699200400021331. PMid:16393798.

5. Liker M, Horga D, Mildner V. Electropalatographic specification of Croatian fricatives /s/ and /z/. Clin Linguist Phon. 2012;26(3):199-215. http://dx.doi.org/10.3109/02699206.2011.602460. PMid:21967279.

6. Liker M, Gibbon FE. Differences in EPG contact dynamics between voiced and voiceless lingual fricatives. J Int Phon Assoc. 2013;43(1):49-64. http:// dx.doi.org/10.1017/S0025100312000436.

7. Liker M, Gibbon FE. Tongue-palate contact timing during $/ \mathrm{s} /$ and $/ \mathrm{z} /$ in English. Phonetica. 2018;75(2):110-31. http://dx.doi.org/10.1159/000479880. PMid:29433122.

8. Dart SN. Articulatory and Acoustic properties of apical and laminal articulations. UCLA WPP. 1991;79:1-155.

9. Dart SN. Comparing French and English coronal consonant articulation. J Phonetics. 1998;26(1):71-94. http://dx.doi.org/10.1006/jpho.1997.0060.

10. Raver-Lampman G, Toreno F, Bing J. An acceptable non-alveolar Japanese /s/. J Second Lang Pronunciation. 2015;1(2):238-53. http://dx.doi.org/10.1075/ jslp.1.2.05rav.

11. Stone M, Gomez AD, Zhuo J, Tchouaga AL, Prince JL. Quantifying tongue tip shape in apical and laminal /s/: contributions of palate shape. J Speech Lang Hear Res. 2019;62(9):3149-59. http://dx.doi.org/10.1044/2019_ JSLHR-S-19-0114. PMid:31469967.

12. Lima FLCN, Silva CEED, Silva LMD, Vassoler AMDO, Fabbron EMG, Berti LC. Análise Ultrassonográfica das líquidas alveolares e fricativas coronais: julgamento de juízes experientes e não experientes. Rev CEFAC. 2018;20(4):422-31. http://dx.doi.org/10.1590/1982-0216201820412317.

13. Raver-Lampman G, Dossou J. Struggling with sibilants: do english speakers use acceptable alternative tongue placements to ronounce $/ \mathrm{s} /$ and $/ \mathrm{z} /$ ? SinoUS English Teaching. 2011;8(6):390-7.

14. Van Borsel J, Van Rentergem S, Verhaeghe L. The prevalence of lisping in young adults. J Commun Disord. 2007;40(6):493-502. http://dx.doi. org/10.1016/j.jcomdis.2006.12.001. PMid:17223125.

15. Martinelli RLC, Fornaro ÉF, Oliveira CJMD, Ferreira LMDB, Rehder MIBC. Correlações entre alterações de fala, respiração oral, dentição e oclusão. Rev CEFAC. 2011;13(1):17-26. http://dx.doi.org/10.1590/S151618462010005000127.

16. Marchesan IQ. Sons da fala e marcadores individuais. In: Redher MI, Cazumbá LF, Cazumbá M, editores. Identificação de falantes. Rio de Janeiro: Revinter; 2015. p. 103-17.

17. Marchesan IQ, Martinelli RLC. Fala: reflexões sobre a prática clínica. In: Silva HJ, Tessitore A, Motta AR, Cunha DA, Berretin-Felix G, Marchesan IQ, editores. Tratado de motricidade orofacial. São José dos Campos: Pulso Editorial; 2019. p. 547-57.

18. Leavy KM, Cisneros GJ, Leblanc EM. Malocclusion and its relationship to speech sound production: redefining the effect of malocclusal traits on sound production. Am J Orthod Dentofacial Orthop. 2016;150(1):116-23. http://dx.doi.org/10.1016/j.ajodo.2015.12.015. PMid:27364213.

19. Genaro KF, Berretin-Felix G, Rehder MIBC, Marchesan IQ. Avaliação miofuncional orofacial: protocolo MBGR. Rev CEFAC. 2009;11(2):23755. http://dx.doi.org/10.1590/S1516-18462009000200009.

20. Rahal A, Motta AR, Fernandes CG, Cunha DA, Migliorucci RR, BerretinFélix G. Manual de motricidade orofacial. 1. ed. São José dos Campos: Pulso Editorial; 2014.

21. Cardoso AFR, Bommarito S, Chiari BM, Motta AR. A confiabilidade da informação fornecida pelo indivíduo a respeito de seu posicionamento habitual de língua. Rev CEFAC. 2011;13(2):236-44. http://dx.doi.org/10.1590/ S1516-18462010005000125.

22. Valentim AF, Furlan RMMM, Perilo TVDC, Motta AR, Casas EBDL. Relação entre a percepção da posição de língua pelo indivíduo e medidas de força da língua nos dentes. CoDAS. 2016;28(5):546-50. http://dx.doi. org/10.1590/2317-1782/20162015256. PMid:27683827.

23. Felício CMD. Fala: um índice sobre adaptação às próteses totais. Pro Fono. 1998;10(1):66-72.

24. Felício CM, Bortolin J, Mazzetto MO, Kriumas R. Efeitos de mudanças no ambiente oral na fala de acordo com a percepção dos falantes. Rev Soc Bras Fonoaudiol. 2005;10(1):7-11.

25. Klein D, Watkins KE, Zatorre RJ, Milner B. Word and nonword repetition in bilingual subjects: a PET study. Hum Brain Mapp. 2006;27(2):153-61. http://dx.doi.org/10.1002/hbm.20174. PMid:16035048.

\section{Contribuiçãa dos autores}

ACVA pesquisador principal, elaboração da pesquisa, elaboração do cronograma, levantamento da literatura, coleta e análise dos dados, redação do artigo; JAG colaboração na elaboração da pesquisa, coleta e análise dos dados e redação do artigo; LLR colaboração na elaboração da pesquisa, análise dos dados e redação do artigo; KFG colaboração na análise dos dados e redação da pesquisa; DAS colaboração na coleta, levantamento da literatura e análise dos dados; EFBC colaboração na análise do desenho do estudo e interpretação dos dados; VCCM elaboração, análise dos dados, redação da pesquisa, correção da redação do artigo, aprovação da versão final, submissão e trâmites do artigo. 